\title{
Exact Solution of the Muñoz-Eaton Model for Protein Folding
}

\author{
Pierpaolo Bruscolini* and Alessandro Pelizzola ${ }^{\dagger}$ \\ Dipartimento di Fisica \& INFM, Politecnico di Torino, c.so Duca degli Abruzzi 24, I-10129 Torino
}

(Dated: October 23, 2018)

\begin{abstract}
A transfer-matrix formalism is introduced to evaluate exactly the partition function of the MuñozEaton model, relating the folding kinetics of proteins of known structure to their thermodynamics and topology. This technique can be used for a generic protein, for any choice of the energy and entropy parameters, and in principle allows the model to be used as a first tool to characterize the dynamics of a protein of known native state and equilibrium population. Applications to a $\beta$-hairpin and to protein CI-2, with comparisons to previous results, are also shown.
\end{abstract}

Recent experimental findings on the folding of small proteins suggest that, despite the complex microscopical dynamics of the proteins in solution, the overall characteristics of the folding kinetics (e.g, the equilibrium rates) could be quite simple, and principally related to the topology of the native state. This has led to the construction of some simple models [1, 2, 3, 4, 5] that, basing on the knowledge of the native fold, aim to predict the kinetic properties and their changes upon mutations.

Even if, usually, the interest in the folding is related to prediction of the native structure, the understanding of the folding process of proteins of known structure is nonetheless of great importance, both for theoretical reasons, and because important diseases are due to the aggregation of partially folded or misfolded proteins [6].

The importance of these models is that they are simple enough to be dealt with quite easily, and have proved to provide relevant information on the folding process as a whole at physiological temperatures. They are useful to test the hypothesis that a reaction coordinate exists, with one or a few major rate-limiting steps, and that motion along it is ruled by the formation of just the native contacts, so that all the others can be effectively averaged out. So, in a sense, they are complementary of the more realistic all-atoms models, that, due to their computational cost, can be applied to characterize just a small part of the folding process at room temperature, or to follow the unfolding at unrealistically high temperatures from a biological point of view.

In this letter we focus on the Muñoz-Eaton (ME) model [1, [, 8], where a protein is described by a chain of peptide bonds that can live in just two states: native and unfolded. Two residues can interact only if they do so in the native structure and if all the intervening residues in the chain between them are native.

In the application to several proteins [8], the authors do not consider a detailed dynamics, but rather relate the relaxation rates to the free energy profiles $F_{j}$ (i.e. the free energy as a function of the number of native bonds $j$ ). Moreover, to reduce the huge number of configurations that any protein can live in, even after the discretization of the states, they calculate these profiles resorting to the "single/double/triple sequence" approximation (SSA/DSA/TSA): they consider only the con- tribution to the free energy of configurations where just one/two/three stretches of native bonds are present, thus effectively lowering the entropy of the unfolded state. Exact solutions of the ME model under the assumption of homogeneous (i.e. residue independent) interactions have been carried out in [5] for the $\beta$-hairpin and the $\alpha$ helix structures, together with a mean field approximation whose accuracy, in comparison with a Monte Carlo simulation, was assessed for protein CI-2.

The main result in this letter is the presentation of a way to drop these approximations and calculate the exact free energy, correlation functions and any other relevant thermodynamical quantity for any given protein, within the model assumptions. The existence of an exact solution is interesting for at least two reasons: the first, is that, in general, exact solutions are rare, and provide useful benchmarks to test approximations and simulations. The second is that, in this case, the solution is also easy to implement, and allows the evaluation of a protein free energy in a few seconds of CPU time. As an illustration, we apply our technique to the $\mathrm{C}$-terminal $\beta$-hairpin of streptococcal protein G B1 and to protein CI2, finding relevant differences from approximate results.

In the ME model [1, 7, 8] the state of a protein of $N+1$ residues can be described by a binary variable $m_{i}$ for each peptide bond $i=1, \ldots, N$ (peptide bond $i$ connects residues $i$ and $i+1$ ). The values $m_{i}=0,1$ indicate that the bond is unfolded or native, respectively. The hamiltonian (indeed a free energy function) reads

$$
H\left(\left\{m_{k}\right\}\right)=\sum_{i=1}^{N-1} \sum_{j=i+1}^{N} \epsilon_{i j} \Delta_{i j} \prod_{k=i}^{j} m_{k}-T \sum_{i=1}^{N} \Delta s_{i} m_{i},
$$

The first term assigns an energy $\epsilon_{i j}<0$ to the contact between bonds $i, j$ (also accounting for the contact between residues $i, j+1)$. This energy gain is present provided that all the bonds from $i$ to $j$ are native, and that the contact exists also in the native structure $\left(\Delta_{i j}=1\right.$ in that case; $\Delta_{i j}=0$ otherwise). The second term represents the entropic cost $\Delta s_{i}<0$ of ordering bond $i$ in the native state. From the above equation, it follows that the contribution $w\left(\left\{m_{k}\right\}\right)=\exp \left[-H\left(\left\{m_{k}\right\}\right) /(R T)\right]$ of any configuration $\left\{m_{k}\right\}=\left(m_{1}, m_{2}, \ldots, m_{N}\right)$ to the partition function $\mathcal{Z}=\sum_{\left\{m_{k}\right\}} w\left(\left\{m_{k}\right\}\right)$ is just the product 


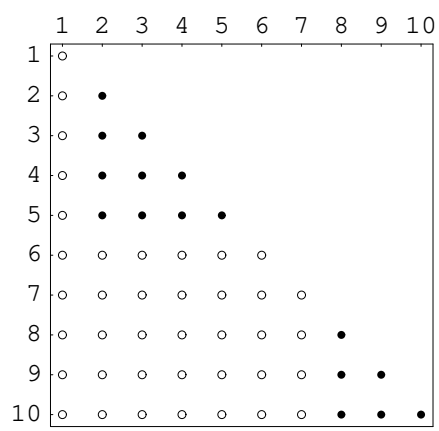

FIG. 1: 2D description of a configuration (i.e $\left\{m_{i}\right\} \equiv$ $\left.\left\{m_{i, i}\right\}=(0,1,1,1,1,0,0,1,1,1)\right)$ of the original model, for $N=10$. Filled symbols represent 1's; empty symbols 0's. Notice that, in any configuration, the 1's will group in triangular regions close to the diagonal, due to Eq. (3). Hence, in each row $j$, all the 1's will stay on the right, yielding just $j+1$ possible states for the row. In evaluating the transfer matrix, the square angle vertex of the triangle, $(j, i)$ (here $(5,2)$ and $(10,8))$, will be attributed the weight $w_{j, i}$.

of the weights of the stretches of native bonds contained in that configuration; namely:

$$
w_{j, i}=\exp \left[-\frac{1}{R T}\left(\sum_{k=i}^{j-1} \sum_{l=k+1}^{j} \epsilon_{k l} \Delta_{k l}-T \sum_{k=i}^{j} \Delta s_{k}\right)\right]
$$

for a native stretch going from bond $i$ to bond $j(i \leq j)$; for later use we define $w_{j+1}=1$.

We start by mapping the original one-dimensional (1D) model, with long range interactions, to a $2 \mathrm{D}$ one, with nearest-neighbour interactions. We do so by introducing

$$
m_{j, i}=\prod_{k=i}^{j} m_{k}
$$

whence the obvious constraints $m_{j, i}=0,1$ and $m_{j, i}=$ $m_{j-1, i} m_{j, i+1}$ follow. The $2 \mathrm{D}$ representation of a generic configuration of the original model is reported in Fig. 1. It can be shown that, in the new variables, it is possible to apply the Cluster Variation Method [9] to write a variational free energy function, whose minimum provides the exact free energy of the model [10]. Here we follow an alternative route, and notice that the states of row $j$ below the diagonal in the 2D model are completely defined specifying the number of 1 's in the row. Notice in fact that all the 1's stay necessarily at the right end of the row (Fig. 1): this is the key observation, allowing the introduction of an efficient transfer matrix formalism to solve the model. In fact, while the diagonal (i.e. the set of original variables of the model) can assume $2^{N}$ configurations, row $j$ in the lattice (but the same is also true for the columns) can assume only $j+1$ states, according to how many 1's are present. Let $\boldsymbol{v}_{k}^{j}$ represent the state with $k 1$ 's $(k=0, \ldots, j)$ of row $j$. The transfer matrix from row $j+1$ to row $j$ will be defined by its action on the vectors $\boldsymbol{v}_{k}^{j+1}$ by the equation:

$$
\begin{aligned}
& Q_{j+1}^{j}(\lambda) \boldsymbol{v}_{k}^{j+1}=\boldsymbol{v}_{k-1}^{j}, \text { for } k=1, \ldots, j+1 \\
& Q_{j+1}^{j}(\lambda) \boldsymbol{v}_{0}^{j+1}=\sum_{k=0}^{j} \lambda^{k} w_{j, j+1-k} \boldsymbol{v}_{k}^{j}
\end{aligned}
$$

where $\lambda$ is a dummy variable whose exponent takes into account the number of 1's (and hence, of native residues) being introduced at row $j$. This will serve to generate the free energy profiles at fixed number of native residues, as explained below. In components, observing that, for each $j, \boldsymbol{v}_{k}^{j}(k=0, \ldots, j)$ can be considered the basis of a $(j+1)$-dimensional space, we write $\left(\boldsymbol{v}_{k}^{j}\right)_{l}=\delta_{l, k+1}$ and

$$
\left(Q_{j+1}^{j}(\lambda)\right)_{l, m}=\left(1-\delta_{m, 1}\right) \delta_{l, m-1}+\delta_{m, 1} \lambda^{l-1} w_{j, j+2-l}
$$

where $l=1, \ldots, j+1$ and $m=1, \ldots, j+2$. Thus, $Q_{j+1}^{j}$ are rectangular matrices; the biggest one, $Q_{N+1}^{N}$, is an $(N+1) \times(N+2)$ matrix.

The partition function of the model will be calculated as $\mathcal{Z} \equiv \mathcal{Z}(\lambda=1)$, where

$$
\mathcal{Z}(\lambda)=\boldsymbol{v}_{0}^{0} \Pi_{N+1}^{0}(\lambda) \boldsymbol{v}_{0}^{N+1}=\sum_{j=0}^{N} Z_{j} \lambda^{j}
$$

Here and below $\Pi_{j}^{i}(\lambda) \equiv Q_{i+1}^{i}(\lambda) \ldots Q_{j}^{j-1}(\lambda)$. From the above expressions it is clear that $\mathcal{Z}(\lambda)$ is the generating function for the contributions $Z_{j}$ coming from the configurations with fixed number of native bonds $j$ : from here we recover the free energy as a function of the native bonds as

$$
F_{j}=-R T \log Z_{j}
$$

In the same fashion it is possible to evaluate the average values of $m_{j, i}$, Eq. (3): introducing the matrix $P_{i, j}$ :

$$
\begin{cases}P_{i, j} \boldsymbol{v}_{k}^{j}=0 & \text { for } k<j-i+1 \\ P_{i, j} \boldsymbol{v}_{k}^{j}=\boldsymbol{v}_{k}^{j} & \text { otherwise }\end{cases}
$$

we will have

$$
\left\langle m_{j, i}\right\rangle=\frac{1}{\mathcal{Z}} \boldsymbol{v}_{0}^{0} \Pi_{j}^{0}(1) P_{i, j} \Pi_{N+1}^{j}(1) \boldsymbol{v}_{0}^{N+1} .
$$

Since $m_{j, i}=1$ represents a native stretch going from $i$ to $j,\left\langle m_{j, i}\right\rangle$ is the probability of observing such a stretch. Other important quantities in defining the folding pathways are the $\mu_{j, i}=\left\langle\left(1-m_{i-1}\right)\left(\prod_{k=i}^{j} m_{k}\right)\left(1-m_{j+1}\right)\right\rangle$, which represent the probability of a native stretch going from bonds $i$ to $j$, preceded and followed by a non-native bond. They can be calculated as

$$
\mu_{j, i}=\frac{1}{\mathcal{Z}} \boldsymbol{v}_{0}^{0} \Pi_{j}^{0} E_{i, j} Q_{j+1}^{j} O_{j+1, j+1} \Pi_{N+1}^{j+1} \boldsymbol{v}_{0}^{N+1},
$$




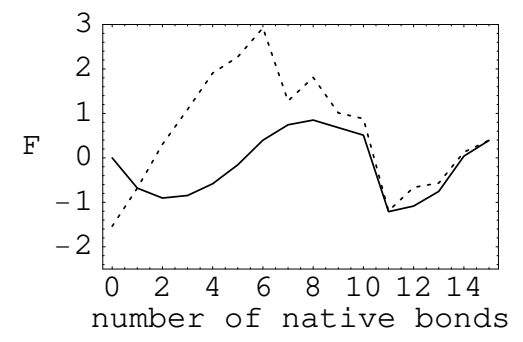

FIG. 2: Free energy profiles for the hairpin according to Eq. (7): x-axis: number of native bonds; y-axis: contribution to free energy (in Kcal/mol). Exact solution (solid line); modified SSA (dotted line; see [-] for details). Here $T=297$ $\mathrm{K}, \Delta H_{h b}=-1.09 \mathrm{Kcal} / \mathrm{mol}, \Delta G_{s c}=-2.03 \mathrm{Kcal} / \mathrm{mol}$, $\Delta S_{\text {conf }}=-3.12 \mathrm{cal} /(\mathrm{K} \mathrm{mol})$, see text. Notice that the native state minimum corresponds to just eleven native bonds: the native state is such that the hydrophobic cluster is formed, but the terminal residues are disordered.

where $Q_{j}^{j-1} \equiv Q_{j}^{j-1}(\lambda=1)$; the matrix $E_{i, j}$ propagates only the state with a stretch of $j-i+1$ native bonds between $i$ and $j$ :

$$
E_{i, j} \boldsymbol{v}_{k}^{j}=\delta_{k, j-i+1} \boldsymbol{v}_{j-i+1}^{j},
$$

while $O_{j, j}$ forces bond $j$ to be non native:

$$
O_{j, j} \boldsymbol{v}_{k}^{j}=\delta_{k, 0} \boldsymbol{v}_{0}^{j} .
$$

We have applied our technique to the $16 \mathrm{C}$-terminal residues from protein $\mathrm{G} \mathrm{B1}$, folding in a $\beta$-hairpin [1], 7 . In order to compare our results with those in [7], we set the contact matrix $\Delta_{i j}$ in Eq. (1) according to Fig. 1 in [7], and specialize $\Delta s_{i}$ and $\epsilon_{i j}$ to the values $\Delta s_{i}=$ $\Delta S_{\text {conf }}$ and $\epsilon_{i j}=p \Delta H_{h b}+q \Delta G_{s c}$, where $p$ is the number of backbone-backbone hydrogen bonds between peptide bonds $i$ and $j$, and $q$ is the number of side-chain hydrophobic interactions between residue $i$ and $j+1$; $\Delta H_{h b}, \Delta G_{s c}$ are the (free) energy contributions of hydrogen bonds and "hydrophobic" interactions, respectively, while $\Delta S_{\text {conf }}$ is the entropy loss upon fixing a bond in its native conformation (refer to the original article for a detailed discussion). We fix $\Delta H_{h b}, \Delta G_{s c}, \Delta S_{\text {conf }}$ by fitting $\left\langle m_{13,3}\right\rangle$, the population of the stretch with formed hydrophobic cluster, to the experimental data in [7], Fig. 3. We find that the exact solution Eq. (7) yields free energy profiles which are smoother than those obtained with SSA, with considerably lower barriers (see Fig. 2). As expected, the differences are greater in the unfolded part of the profile, where the entropic contribution of partially ordered conformations dominates. Even if we do not attempt a microscopic dynamics, upon assuming that a single-residue dynamics in the equilibrium landscape may represent the true kinetics we can try to characterize the (equilibrium) folding barriers. To this end, we consider the values of the $\mu_{j, i}$ of Eq. (10). They allow to follow the kinetic pathway of the hairpin, since the length of the native stretches must increase on approaching the native

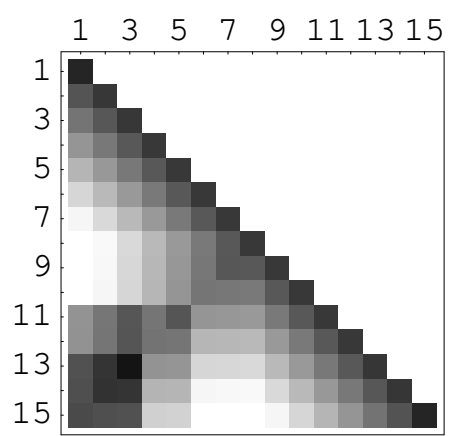

FIG. 3: Density plot of $-\log \mu_{j, i}$ (see Eq. (10)) for the hairpin: darker regions correspond to more probable stretches. It is possible to recognize the unfolding pathway as $(13,3)-$ $(12,3)-(11,3)-(11,4)-(11,5)-((10,5)$ or $(11,6))-(10,6)-$ $((9,6)$ or $(10,7))-(9,7)$, where the position $(j, i)$ in the matrix corresponds to a native stretch from bond $i$ to $j>i$.

state, and it can be verified that, for the hairpin, it is most likely that a $n$-bonds native stretch is created by adding a native site at one end of $(n-1)$-stretch, rather than filling a 1-bond gap between two shorter stretches and merging them. Fig. 3 suggests that the barrier in the folding/unfolding pathway corresponds to the formation of six-bond-long stretches, either from peptide bond 5 (Y45-D46) to 10 (K50-T51) or from 6 (D46-D47) to 11 (T51-F52). The presence of a folding barrier thus appears as a feature of the model, and not just of SSA, and contrasts with the results in 11 .

Coming to proteins, we have applied our exact solution to the 65 terminal residues of protein CI-2 (2CI2), choosing the values of $\epsilon_{i j} \Delta_{i j}$ in Eq. (11) according to the following procedure. As in [8], we define an atomic contact to be present if two nonhydrogen atoms, from residues $i$ and $j>i+2$, are closer than $0.4 \mathrm{~nm}$ and we give an energy $k \epsilon$ to residues contacts involving $5(k-1)<n_{a t} \leq 5 k$ atomic contacts. Moreover, we get the native secondary structure from 12 and assign to the peptide bond preceding a residue marked by $\mathrm{B}, \mathrm{E}, \mathrm{G}, \mathrm{H}, \mathrm{I}, \mathrm{T}$, the entropy cost $\Delta s_{1}$ of the "structured" elements, while the other symbols will get the entropy $\Delta s_{0}$ of the "less structured" parts (i.e. coils, loops). Then, to fix $\epsilon, \Delta s_{1}, \Delta s_{0}$ we assume that the protein has a two-state thermodynamics: the equilibrium population of the native state will be given by $\theta=\mathcal{Z}_{\text {nat }} / \mathcal{Z}$, where $\mathcal{Z}_{\text {nat }}$ is the sum of the contributions from the right of the barrier in Fig. A Then, we fit the calorimetric data for $\Delta G=G_{u}-G_{n}$ in [13], by asking that $(1-\theta) / \theta=\exp (-\Delta G / R T)$.

We can then study the differences between the SSA/DSA and the exact free energy profiles, as well as the equilibrium values for the $\mu_{j, i}$ of Eq. (10). The results are reported in Figs. 1 and 5 respectively, at the temperature of equal native and unfolded population $T=343.54$ $\mathrm{K}$ 13]. It is clear that SSA and DSA deeply underestimate the configurational entropy of the unfolded state, 


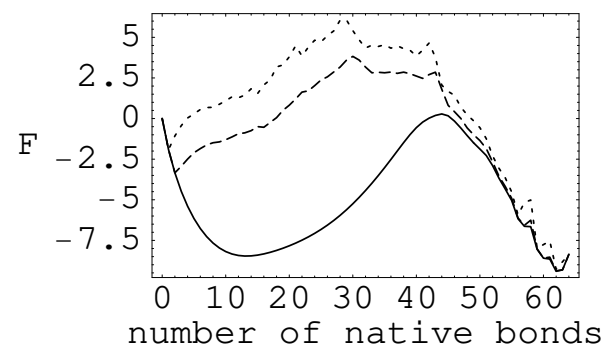

FIG. 4: Free energy profiles for protein CI-2 according to Eq. (7): x-axis: number of native bonds; y-axis: contribution to free energy (in $\mathrm{Kcal} / \mathrm{mol}$ ). Exact solution (solid line); SSA (dotted); DSA (dashed); see 11 for details. Here $\epsilon=-0.550$ $\mathrm{Kcal} / \mathrm{mol}, \Delta s_{0}=-1.327 \mathrm{cal} /(\mathrm{K} \mathrm{mol}), \Delta s_{1}=-3.863 \mathrm{cal} /(\mathrm{K}$ $\mathrm{mol})$, from the fit of calorimetric data: see text.

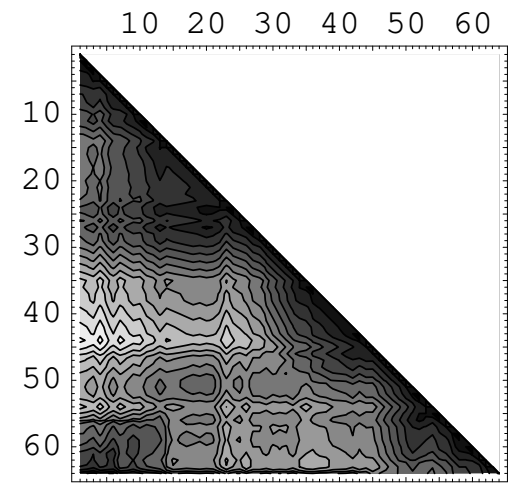

FIG. 5: Contour plot of $-\log \mu_{j, i}$ (Eq. (10) for CI-2: darker regions represent more likely stretches. Parameters as before.

yielding different barriers to folding and unfolding. The exact free energy profile is also much smoother than the

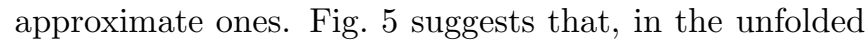
state, the helix (13-24) and the region including a turn (51-56) are the most populated. Experimentally, the unfolded state is devoid of fixed structure [14], but the Nterminal part of the helix, together with the residues L50, I58 15], constitute the folding nucleus: the model gives interesting clues about this, even if with a turn overestimation [16]. The helix was already noticed to be particularly populated in [2, 5, 17.

From Fig. [5 we can also speculate about the folding pathway: assuming an equilibrium folding dynamics, with the number of native bonds increasing of at most one bond per time step, we try to characterize the unfolding pathway by asking whether a $\mu_{j, i}$ will more likely drop an end, producing $\mu_{j-1, i}$ or $\mu_{j, i+1}$, or will split in two stretches $(i, k-1)$ and $(k+1, j)$ for some $k$. Upon reverting the unfolding pathway, the above analysis suggests that the folding starts with the formation of the turn at 54-55; it grows up to the last residue and then towards the N-terminus; that folds last. Although this result correctly suggests that the helix alone cannot nucleate the folding [2, 14, this pathway does not agree with the experimental one: this could be partially due to our one-residue "dynamics"; notice though that the model cannot account for interactions as those in the folding nucleus, involving residues that are not in the same native stretch: this is probably its major limit.

In conclusion, we have presented the complete exact solution for the thermodynamics of the ME model, in a form which is also easy to implement and to apply to real proteins. We have analyzed the cases of a $\beta$-hairpin peptide and of protein CI-2, finding in both cases relevant differences from previously published results. Our solution opens a wide range of applications to real proteins, especially after a better characterization of the relationship between equilibrium properties and folding dynamics is found: work is in progress along these lines.

We are grateful to A. Maritan, F. Cecconi and A. Flammini for drawing our attention to the ME model, for communicating their results prior to publication and for fruitful discussions. We are also indebted to M. Vendruscolo for kindly sharing with us his computer program to calculate contact maps.

* Electronic address: pbr@athena.polito.it

$\dagger$ Electronic address: alex@athena.polito.it

[1] V. Muñoz, P. A. Thompson, J. Hofrichter and W. A. Eaton, Nature (London) 390, 196 (1997).

[2] E. Alm and D. Baker, Proc. Natl. Acad. Sci. U.S.A. 96, 11305 (1999).

[3] O. V. Galzitskaya and A. V. Finkelstein, Proc. Natl. Acad. Sci. U.S.A. 96, 11299 (1999).

[4] D. Baker, Nature (London) 405, 39 (2000).

[5] A. Flammini, J. R. Banavar and A. Maritan, Europhys. Lett., 58, 623 (2002).

[6] P. T. Landsbury Jr., Proc. Natl. Acad. Sci. U.S.A. 96, 3342 (1999).

[7] V. Muñoz, E. R. Henry, J. Hofrichter and W. A. Eaton, Proc. Natl. Acad. Sci. U.S.A. 95, 5872 (1998).

[8] V. Muñoz and W. A. Eaton, Proc. Natl. Acad. Sci. U.S.A. 96, 11311 (1999).

[9] see the volume Prog. Theor. Phys. Suppl. 115, 1 (1994).

[10] P. Bruscolini and A. Pelizzola, unpublished results.

[11] A. R. Dinner, T. Lazaridis, and M. Karplus, Proc. Natl. Acad. Sci. U.S.A. 96, 9068 (1999).

[12] http://www.rcsb.org/pdb/index.htm

[13] G. I. Makhatadze and P. L. Privalov, Adv. Prot. Chem. 47, 307 (1995).

[14] A. R. Fersht, Proc. Natl. Acad. Sci. U.S.A. 92, 10869 (1995).

[15] In Ref. [14] Fersht studied the 64-terminal-residues fragment; the residues' number here is shifted by one.

[16] Notice anyway that the greater stability of the turn, and of the helix C-terminus over the $\mathrm{N}$ one, could be an artefact of dividing residues according to secondary structure: the presence in those regions of "less structured" residues K25, D53, D56 entails a smaller cost $\Delta s_{0}$ upon folding.

[17] C. Micheletti, J. R. Banavar, A. Maritan and F. Seno, Phys. Rev. Lett. 82, 3372 (1999). 